\title{
Spectral Properties of Two-slanted Matrices
}

\author{
Lothar Berg and Gerlind Plonka
}

\begin{abstract}
For two-slanted matrices, there is shown the close connection between their spectral properties and the zeros of their corresponding symbols. The results are applied to two-scale difference equations.
\end{abstract}

Subject Classification: 15A18, 39B22

Key words: Two-slanted matrices, spectral properties, two-scale difference equations

\section{Introduction}

For a given finite set of real or complex numbers $c_{0}, \ldots, c_{n}$ with $c_{0} c_{n} \neq 0$ we consider the twoslanted matrix $A=\left(c_{2 j-k}\right), j, k=0, \ldots, n$ (where $c_{j}=0$ for $j<0$ and $j>n$, respectively), i.e.,

$$
A=\left[\begin{array}{ccccc}
c_{0} & 0 & 0 & \ldots & 0 \\
c_{2} & c_{1} & c_{0} & \ldots & 0 \\
\vdots & & \ddots & & \vdots \\
0 & \ldots & c_{n} & c_{n-1} & c_{n-2} \\
0 & \ldots & 0 & 0 & c_{n}
\end{array}\right]
$$

The matrix $A$ can completely be described by its symbol

$$
P(z)=\frac{1}{2} \sum_{\nu=0}^{n} c_{\nu} z^{\nu} .
$$

Further we shall consider the infinite two-slanted matrix $\boldsymbol{A}=\left(c_{2 j-k}\right), j, k=0,1,2 \ldots$, which is very useful for the study of spectral properties of $A$. Indeed, the first $n+1$ entries of a left eigenvector $\boldsymbol{w}^{\mathrm{T}}=\left(w_{0}, w_{1}, \ldots\right)$ of $\boldsymbol{A}$ form a left eigenvector $w^{\mathrm{T}}$ of $A$ to the same eigenvalue, and, vice versa, every left eigenvector $w^{\mathrm{T}}=\left(w_{0}, w_{1}, \ldots, w_{n}\right)$ of $(1)$ can be continued to an eigenvector $\boldsymbol{w}^{\mathrm{T}}$ of $\boldsymbol{A}$.

Two-slanted matrices play an important role for the solution of two-scale difference equations of the form

$$
\varphi\left(\frac{t}{2}\right)=\sum_{\nu=0}^{n} c_{\nu} \varphi(t-\nu),
$$

$t \in \mathbb{R}$ (see e.g. $[1,2,3,5,6]$ ). In this context, the symbol $P(z)$ is the so-called two-scale symbol (or refinement mask) of the equation (3).

The purpose of this paper is to enlighten some connections between zeros of the symbol $P(z)$ on the unit circle and spectral properties of $A$ and $\boldsymbol{A}$, respectively. 
In the second part of the paper, these results are applied to solutions of two-scale difference equations. We shall generalize Theorem 4.1 in [1], concerning the nonexistence of proper root vectors of $A$ to the eigenvalue 1 , if (3) possesses a nontrivial, continuous solution $\varphi$. Further, we present new methods for determining the vector $\psi(0)=(\varphi(0), \ldots \varphi(n))^{\mathrm{T}}$, if $A$ possesses a multiple eigenvalue 1.

\section{Roots of unity}

Let $\mathcal{M}$ be a finite set of complex numbers different from zero, which is closed under the mapping $z \mapsto z^{2}$. Then, for every $\zeta \in \mathcal{M}$, there exist two integers $\sigma, \tau$ with $0 \leq \sigma<\tau$, such that $\zeta^{2^{\sigma}}=\zeta^{2^{\tau}}$. Hence, all elements of $\mathcal{M}$ are roots of unity in view of $\zeta^{2^{\sigma}-2^{\tau}}=1$.

If we choose $\sigma$ and $\tau$ minimal, we can say that $\zeta$ generates a discrete flow $\zeta^{2^{\nu}}, \nu=0,1,2, \ldots$, with a preperiod of length $\sigma$ and a cycle of length $m=\tau-\sigma$, cf. [9]. The length of the preperiod can be zero, and the length of the cycle can be 1 . The last property occurs for $\zeta=1$.

Example 1. For $\mathcal{M}=\left\{e^{\pi i / 6}, e^{\pi i / 3}, e^{2 \pi i / 3}, e^{4 \pi i / 3}\right\}$ and $\zeta=e^{\pi i / 6}$ we find $\sigma=2, \tau=4$, i.e., $\zeta$ generates a preperiod of length $\sigma=2$ and a cycle $\left\{e^{2 \pi i / 3}, e^{4 \pi i / 3}\right\}$ of length $m=2$.

A subset of $\mathcal{M}$ is called an orbit of $\mathcal{M}$, if all elements of the subset generate the same cycle. This means that different orbits are disjunct, and every orbit has only one cycle, cf. [12].

For the present, we assume that the set $\mathcal{M}$ consists of one orbit only. Though all squares of the elements of $\mathcal{M}$ are lying in $\mathcal{M}$, not all corresponding square roots do it, hence we can split $\mathcal{M}$ into three disjunct subsets $\mathcal{M}_{\mu}, \mu=0,1,2$, in the following way: We define $\mathcal{M}_{\mu}$ as the subset of all elements of $\mathcal{M}$ possessing exactly $\mu$ square roots in $\mathcal{M}$. In Example 1, we find $\mathcal{M}_{0}=\left\{e^{\pi i / 6}\right\}, \mathcal{M}_{1}=\left\{e^{\pi i / 3}, e^{4 \pi i / 3}\right\}, \mathcal{M}_{2}=\left\{e^{2 \pi i / 3}\right\}$.

Next the signs of the considered square roots must be fixed. For the elements $\zeta$ of $\mathcal{M}_{1}$, we denote the square roots lying in $\mathcal{M}$ by $\sqrt{\zeta}$. For the square roots of the elements of $\mathcal{M}_{2}$, we fix the signs as follows: If $\zeta$ belongs to the cycle, then $\sqrt{\zeta}$ shall also belong to the cycle, otherwise, we choose the signs arbitrarily. Now, we number the elements of $\mathcal{M}$ in the following way: We denote the elements of the cycle by $\zeta_{1}, \ldots, \zeta_{m}$ in such a way that $\zeta_{\nu}^{2}=\zeta_{\nu-1}$ for $\nu=2, \ldots, m$ and $\zeta_{1}^{2}=\zeta_{m}$. Having already numbered the elements of a strict subset of $\mathcal{M}$, we choose an element $\zeta$ so that $\zeta^{2}$ is already numbered, give to it the next number, and the consecutive numbers to the consecutive square roots without minus-sign, as long as they belong to $\mathcal{M}$.

If we proceed in this way up to the last element, we have the following relations:

$$
\begin{array}{ll}
\sqrt{\zeta_{\nu}}=\zeta_{\nu+1} & \text { for } \zeta_{\nu} \in \mathcal{M}_{1} \cup \mathcal{M}_{2} \text { and } \nu \neq m, \sqrt{\zeta_{m}}=\zeta_{1}, \\
\pm \sqrt{\zeta_{\nu}} \notin \mathcal{M} & \text { for } \zeta_{\nu} \in \mathcal{M}_{0}, \\
-\sqrt{\zeta_{\nu}} \notin \mathcal{M} & \text { for } \zeta_{\nu} \in \mathcal{M}_{1}, \\
-\sqrt{\zeta_{\nu}}=\zeta_{n_{\nu}} & \text { for } \zeta_{\nu} \in \mathcal{M}_{2} \text { with some } n_{\nu}>\max (m, \nu) .
\end{array}
$$

The first property simplifies, if we denote $\zeta_{m}$ by $\zeta_{0}$ on the left-hand side. In our above Example 1 we could choose for instance $\zeta_{1}=e^{4 \pi i / 3}, \zeta_{2}=e^{2 \pi i / 3}, \zeta_{3}=e^{\pi i / 3}, \zeta_{4}=e^{\pi i / 6}$, so that $\sqrt{\zeta_{1}}=\zeta_{2}, \sqrt{\zeta_{2}}=\zeta_{1}, \sqrt{\zeta_{3}}=\zeta_{4}$ and $-\sqrt{\zeta_{2}}=\zeta_{3}$.

Example 2. Let us consider a more delicate example of a set $\mathcal{M}$ with one orbit only, with cycle of length 4 and several preperiods. Let $\mathcal{M}_{0}=\left\{\zeta_{7}, \zeta_{8}, \zeta_{10}, \zeta_{13}, \zeta_{14}, \zeta_{15}\right\}, \mathcal{M}_{1}=$ 


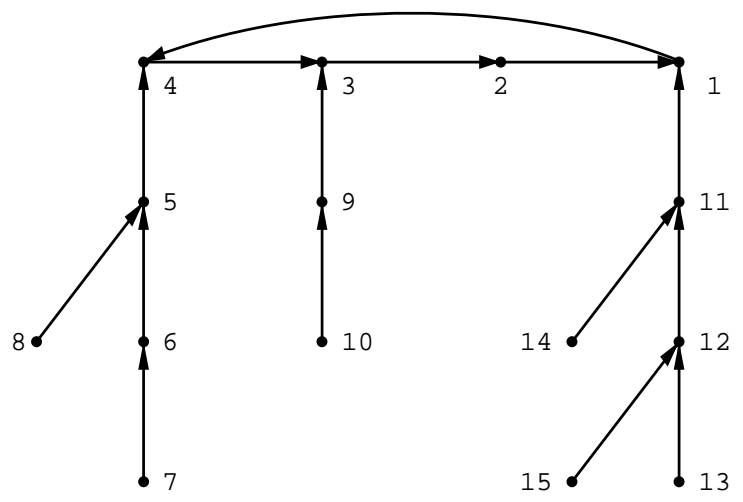

Figure 1: Directed graph of roots of unity for Example 2

$\left\{\zeta_{2}, \zeta_{6}, \zeta_{9}\right\}, \mathcal{M}_{2}=\left\{\zeta_{1}, \zeta_{3}, \zeta_{4}, \zeta_{5}, \zeta_{11}, \zeta_{12}\right\}$ and $n_{1}=11, n_{3}=9, n_{4}=5, n_{5}=8, n_{11}=14, n_{12}=$ 15. In Figure 1, the numbers indicate the indices and the arrows indicate squaring of the elements.

This set $\mathcal{M}$ can e.g. be obtained with $\zeta_{\nu}=e^{r_{\nu} \pi i}$, where

\begin{tabular}{l||llll|lll|l|ll|lll|l|l}
$\nu$ & 1 & 2 & 3 & 4 & 5 & 6 & 7 & 8 & 9 & 10 & 11 & 12 & 13 & 14 & 15 \\
\hline$r_{\nu}$ & $\frac{6}{5}$ & $\frac{8}{5}$ & $\frac{4}{5}$ & $\frac{2}{5}$ & $\frac{1}{5}$ & $\frac{1}{10}$ & $\frac{1}{20}$ & $\frac{11}{10}$ & $\frac{7}{5}$ & $\frac{7}{10}$ & $\frac{3}{5}$ & $\frac{3}{10}$ & $\frac{3}{20}$ & $\frac{13}{10}$ & $\frac{23}{20}$
\end{tabular}.

Let us mention that the power of $\mathcal{M}_{0}$ is the same as the power of $\mathcal{M}_{2}$, since the number of elements being square roots in $\mathcal{M}$ is the same as the number $l$ of all elements of $\mathcal{M}$. Moreover, $l$ is also the number of the remaining square roots (of elements of $\mathcal{M}$ ), being not elements of $\mathcal{M}$, because the number of all square roots of elements of $\mathcal{M}$ is $2 l$. In the following, we denote the remaining square roots (being not contained in $\mathcal{M}$ ) in an arbitrary order by $\omega_{1}, \ldots, \omega_{l}$. Of course, these $\omega_{\nu}$ are also roots of unity. If $\mathcal{M}$ contains an element with a preperiod, then $\mathcal{M}_{0} \neq \emptyset$ and at least for two indices $\mu, \nu$ we have $\omega_{\mu}=-\omega_{\nu}$, i.e., $\left\{\omega_{1}, \ldots, \omega_{l}\right\}$ contains symmetric roots.

Each polynomial $P(z)$ can uniquely be related with a special set $\mathcal{M}_{P}$ as follows. We consider sets $\mathcal{M}$ of (pairwise different) zeros of $P(z)$ being at the same time roots of unity, and form the closure $\overline{\mathcal{M}}$ of $\tilde{\mathcal{M}}$ with respect to the mapping $z \mapsto z^{2}$ as well as the differences $\overline{\mathcal{M}} \backslash \tilde{\mathcal{M}}$. If, among these differences, there exist such which have the same number of elements as the corresponding $\tilde{\mathcal{M}}$, then $\mathcal{M}_{P}$ is defined as the largest of these special differences. Only the case $\mathcal{M}_{P} \neq \emptyset$ is interesting for us.

Example 3. Let $P(z)=\frac{1}{8}(1+i)(z-i)\left(z^{3}+1\right)^{2}$ with the different zeros $i,-1, e^{\pi i / 3}, e^{5 \pi i / 3}$. Choosing $\tilde{\mathcal{M}}=\left\{-1, e^{\pi i / 3}, e^{5 \pi i / 3}\right\}$, we find $\overline{\mathcal{M}}=\left\{-1,1, e^{\pi i / 3}, e^{5 \pi i / 3}, e^{2 \pi i / 3}, e^{4 \pi i / 3}\right\}$. Indeed, $\overline{\mathcal{M}} \backslash \tilde{\mathcal{M}}$ contains 3 elements and $\mathcal{M}_{P}=\overline{\mathcal{M}} \backslash \tilde{\mathcal{M}}=\left\{1, e^{2 \pi i / 3}, e^{4 \pi i / 3}\right\}$ is maximal.

\section{Left eigenvectors of $A$}

With the foregoing notations and assumptions, we find:

Theorem 1 Let $P(z)$ in (2) be the symbol of a two-slanted matrix $A$. Further, let $\mathcal{M}=$ $\left\{\zeta_{1}, \ldots, \zeta_{l}\right\}$ be a set of roots of unity being closed under the mapping $z \mapsto z^{2}$, consisting of 
one orbit only, and being denoted as in Section 2. Assume that $P\left(\omega_{\nu}\right)=0$ for $\nu=1, \ldots, l$, i.e. $\mathcal{M} \subseteq \mathcal{M}_{P}$, and $P\left(\zeta_{\mu}\right) \neq 0$ for $\mu=1, \ldots, m$, where $m$ is the length of the cycle. Then $A$ has $m$ eigenvalues $\lambda, \lambda \varepsilon, \ldots, \lambda \varepsilon^{m-1}$, where $\varepsilon=e^{2 \pi i / m}$, and $\lambda$ is a special solution of

$$
\lambda^{m}=\prod_{\mu=1}^{m} P\left(\zeta_{\mu}\right)
$$

If additionally $P^{\prime}\left(\omega_{\nu}\right)=P^{\prime \prime}\left(\omega_{\nu}\right)=\ldots=P^{(r)}\left(\omega_{\nu}\right)=0$ for a fixed $r \in \mathbb{N}$ and $\nu=1, \ldots$, then the mr numbers

$$
\frac{\lambda}{2} \varepsilon^{\mu-1}, \frac{\lambda}{4} \varepsilon^{\mu-1}, \ldots, \frac{\lambda}{2^{r}} \varepsilon^{\mu-1}
$$

with $\mu=1, \ldots, m$, are also eigenvalues of $A$.

Proof. We carry out the proof for $\boldsymbol{A}$ instead of $A$. For the components of a left eigenvector $\boldsymbol{w}^{\mathrm{T}}=\left(w_{j}\right)_{j \geq 0}$ of $\boldsymbol{A}$, we use the ansatz

$$
w_{j}=\sum_{k=1}^{l} d_{k}(j) \zeta_{k}^{j}
$$

at first with constant coefficients $d_{k}(j)=d_{k} \in \mathbb{C}$ and $\zeta_{k} \in \mathcal{M}$. With the notation $\left(\zeta^{j}\right)^{\mathrm{T}}=$ $\left(1, \zeta, \zeta^{2}, \ldots\right)$, we can verify that

$$
\left(\zeta^{j}\right)^{\mathrm{T}} \boldsymbol{A}=P(\sqrt{\zeta})\left((\sqrt{\zeta})^{j}\right)^{\mathrm{T}}+P(-\sqrt{\zeta})\left((-\sqrt{\zeta})^{j}\right)^{\mathrm{T}},
$$

(see [1], Formula (3.2)) which implies in components

$$
\left(\boldsymbol{w}^{\mathrm{T}} \boldsymbol{A}\right)_{j}=\sum_{k=1}^{l} d_{k}\left(P\left(\sqrt{\zeta_{k}}\right)\left(\sqrt{\zeta_{k}}\right)^{j}+P\left(-\sqrt{\zeta_{k}}\right)\left(-\sqrt{\zeta_{k}}\right)^{j}\right) .
$$

In view of our notations in Section 2, equation $\lambda \boldsymbol{w}^{\mathrm{T}}=\boldsymbol{w}^{\mathrm{T}} \boldsymbol{A}$ reads in components

$$
\lambda \sum_{k=1}^{l} d_{k} \zeta_{k}^{j}=\sum_{\nu \in \mathcal{J}_{1}} d_{\nu} P\left(\zeta_{\nu+1}\right) \zeta_{\nu+1}^{j}+\sum_{\nu \in \mathcal{J}_{2}} d_{\nu} P\left(\zeta_{n_{\nu}}\right) \zeta_{n_{\nu}}^{j},
$$

where $\mathcal{J}_{1}$ is the set of indices $\nu$ with $\zeta_{\nu} \in \mathcal{M}_{1} \cup \mathcal{M}_{2}$ and $\mathcal{J}_{2}$ the set with $\zeta_{\nu} \in \mathcal{M}_{2}$, but with $\zeta_{0}$ instead of $\zeta_{m}$. Comparing the coefficients of $\zeta_{k}$, we obtain a system of equations for the calculation of the coefficients $d_{k}$. In view of $n_{\nu}>\max (\nu, m)$, the matrix of the system has the form

$$
\left[\begin{array}{ll}
B & O \\
C & D
\end{array}\right]
$$


where $O$ is a suitable zero matrix, $D$ is a lower triangular matrix of size $(l-m) \times(l-m)$ with constant diagonal elements $\lambda$, and $B$ is the $m \times m$ matrix

$$
B=\left[\begin{array}{cccccc}
\lambda & 0 & \ldots & \ldots & 0 & -P\left(\zeta_{1}\right) \\
-P\left(\zeta_{2}\right) & \lambda & \ddots & & & 0 \\
0 & -P\left(\zeta_{3}\right) & \lambda & \ddots & & \vdots \\
\vdots & \ddots & \ddots & \ddots & \ddots & \vdots \\
\vdots & & \ddots & \ddots & \lambda & 0 \\
0 & \ldots & \ldots & 0 & -P\left(\zeta_{m}\right) & \lambda
\end{array}\right]
$$

which for $m=1$ must be interpreted as $B=\lambda-P\left(\zeta_{1}\right)$. The determinant of (8) reads

$$
\left(\lambda^{m}-\prod_{\nu=1}^{m} P\left(\zeta_{\nu}\right)\right) \lambda^{l-m}
$$

and vanishes for (4). If this condition is satisfied, the first $m$ equations

$$
\lambda d_{k}=P\left(\zeta_{k}\right) d_{k-1} \quad \text { for } k=1, \ldots, m
$$

with $d_{0}=d_{m} \neq 0$ imply recursively

$$
d_{k}=P\left(\zeta_{k}\right) \ldots P\left(\zeta_{1}\right) \lambda^{-k} d_{0} \quad \text { for } k=1, \ldots, m .
$$

Finally, in view of $\lambda \neq 0$, the remaining equations can also be solved recursively, and we have finished the proof for $r=0$. The eigenvector with the components (5) is unique up to the factor $d_{0}$.

Let now $P^{\prime}\left(\omega_{\nu}\right)=\ldots=P^{(r)}\left(\omega_{\nu}\right)=0$ for a fixed $r \geq 1$. We show the assertion inductively and assume that the theorem is already proved for $r-1$ instead of $r$. Differentiating (6) $\varrho$ times with respect to $\zeta$, we obtain after multiplication by $\frac{\zeta^{\varrho}}{\varrho !}$

$$
\begin{gathered}
\left(\left(\begin{array}{l}
j \\
\varrho
\end{array}\right) \zeta^{j}\right)^{\mathrm{T}} \boldsymbol{A}= \\
\sum_{\nu=0}^{\varrho} \frac{\zeta^{\varrho-\nu}}{(\varrho-\nu) !}\left[(P(\sqrt{\zeta}))^{(\varrho-\nu)}\left(\left(\begin{array}{c}
j / 2 \\
\nu
\end{array}\right)(\sqrt{\zeta})^{j}\right)^{\mathrm{T}}+(P(-\sqrt{\zeta}))^{(\varrho-\nu)}\left(\left(\begin{array}{c}
j / 2 \\
\nu
\end{array}\right)(-\sqrt{\zeta})^{j}\right)^{\mathrm{T}}\right] .
\end{gathered}
$$

Using for $\boldsymbol{w}$ the ansatz (5) with coefficients

$$
d_{k}(j)=\sum_{\varrho=0}^{r} \alpha_{k \varrho}\left(\begin{array}{l}
j \\
\varrho
\end{array}\right),
$$

we find for the $j$-th component of $\boldsymbol{w}^{\mathrm{T}} \boldsymbol{A}$

$$
\begin{gathered}
\left(\boldsymbol{w}^{\mathrm{T}} \boldsymbol{A}\right)_{j}= \\
\sum_{k=1}^{l} \sum_{\varrho=0}^{r} \alpha_{k \ell} \sum_{\sigma=0}^{\varrho} \frac{\zeta_{k}^{\varrho-\sigma}}{(\varrho-\sigma) !}\left(\begin{array}{c}
j / 2 \\
\sigma
\end{array}\right)\left[\left(P\left(\sqrt{\zeta_{k}}\right)\right)^{(\varrho-\sigma)}\left(\sqrt{\zeta_{k}}\right)^{j}+\left(P\left(-\sqrt{\zeta_{k}}\right)\right)^{(\varrho-\sigma)}\left(-\sqrt{\zeta_{k}}\right)^{j}\right] .
\end{gathered}
$$


In view of our assumptions, equation $\frac{\lambda}{2^{r}} \boldsymbol{w}^{T}=\boldsymbol{w}^{\mathrm{T}} \boldsymbol{A}$ reads in components

$$
\begin{aligned}
\frac{\lambda}{2^{r}} \sum_{k=1}^{l} \sum_{\varrho=0}^{r} \alpha_{k \varrho}\left(\begin{array}{l}
j \\
\varrho
\end{array}\right) \zeta_{k}^{j}= & \sum_{\nu \in \mathcal{J}_{1}} \sum_{\varrho=0}^{r} \alpha_{\nu \varrho} \sum_{\sigma=0}^{\varrho} \frac{\zeta_{\nu}^{\varrho-\sigma}}{(\varrho-\sigma) !}\left(\begin{array}{c}
j / 2 \\
\sigma
\end{array}\right)\left(P\left(\zeta_{\nu+1}\right)\right)^{(\varrho-\sigma)} \zeta_{\nu+1}^{j} \\
& +\sum_{\nu \in \mathcal{J}_{2}} \sum_{\varrho=0}^{r} \alpha_{\nu \varrho} \sum_{\sigma=0}^{\varrho} \frac{\zeta_{\nu}^{\varrho-\sigma}}{(\varrho-\sigma) !}\left(\begin{array}{c}
j / 2 \\
\sigma
\end{array}\right) P\left(\zeta_{n_{\nu}}\right) \zeta_{n_{\nu}}^{j}
\end{aligned}
$$

with index sets $\mathcal{J}_{1}$ and $\mathcal{J}_{2}$ as before. Comparing the coefficients of $j^{r}$, we obtain after multiplication by $2^{r} r$ !

$$
\lambda \sum_{k=1}^{l} \alpha_{k r} \zeta_{k}^{j}=\sum_{\nu \in \mathcal{J}_{1}} \alpha_{\nu r} P\left(\zeta_{\nu+1}\right) \zeta_{\nu+1}^{j}+\sum_{\nu \in \mathcal{J}_{2}} \alpha_{\nu r} P\left(\zeta_{n_{\nu}}\right) \zeta_{n_{\nu}}^{j} .
$$

But this is exactly the system (7) only with $\alpha_{k r}$ instead of $d_{k}$.

Hence, choosing $\alpha_{m r}=d_{0}$, we find $\alpha_{k r}=d_{k}$. Having already determined $\alpha_{k \ell}$ for $\varrho=$ $s+1, \ldots, r$, we compare the coefficients of $j^{s}$, and obtain after multiplication by $2^{s} s$ !

$$
\frac{\lambda}{2^{r-s}} \sum_{k=1}^{l} \alpha_{k s} \zeta_{k}^{j}=\sum_{\nu \in \mathcal{J}_{1}} \alpha_{\nu s} P\left(\zeta_{\nu+1}\right) \zeta_{\nu+1}^{j}+\sum_{\nu \in \mathcal{J}_{2}} \alpha_{\nu s} P\left(\zeta_{n_{\nu}}\right) \zeta_{n_{\nu}}^{j}+\ldots
$$

where the dots indicate already known terms. The matrix of the system arises from that of (7) by the replacement $\lambda \mapsto \lambda / 2^{r-s}$. Hence, in view of (4) and (10), its determinant reads

$$
\frac{\lambda^{l}}{2^{(r-s) l}}\left(1-2^{(r-s) m}\right)
$$

and is different from zero. Thus the system is uniquely solvable, and the theorem is proved.

From the proof of Theorem 1, we obtain the following generalization of Lemma 7.1.11 of [4]:

Corollary 1 Let the assumptions of Theorem 1 be satisfied. Then, to the eigenvalues $\frac{\lambda}{2^{s}} \varepsilon^{\nu}$ with $0 \leq s \leq r, 0 \leq \nu<m$ belong left eigenvectors $w^{\mathrm{T}}$ of $A$ with components of the form $(5)$, where $d_{1}(j), \ldots, d_{m}(j)$ have exactly the degree $s$, and the other $d_{k}(j)$ have at most the degree $s$. In the case $s=0$, the components (5) of the corresponding vectors $\boldsymbol{w}^{\mathrm{T}}$ are $\left(2^{m}-1\right)$-periodic.

Remarks. 1. For a fixed cycle $\zeta_{1}, \ldots, \zeta_{m}$ and a corresponding eigenvalue $\lambda$ of $A$ satisfying (4), there can exist only one linearly independent eigenvector $\boldsymbol{w}^{\mathrm{T}}$ with components of the form (5). However, if $\mathcal{M}$ consists of $k$ orbits, then there can exist $k$ linearly independent eigenvectors to the same eigenvalue with components of the form (5). Moreover, every twoslanted matrix (1) has also the eigenvector $(1,0, \ldots, 0)$ to the eigenvalue $c_{0}$, which can also be interpreted as $\left(\zeta^{j}\right)$, but in the exceptional case $\zeta=0$ with the definition $0^{0}=1$.

2. In special cases, we can calculate $\lambda$ in (4) explicitly. Let the symbol $P(z)$ of $A$ be of the form

$$
P(z)=\frac{Q\left(z^{2}\right)}{2 Q(z)}
$$


where $Q(z)$ is a polynomial, and let $\zeta_{1}, \ldots, \zeta_{m}$ be simple zeros of $Q(z)$. Then

$$
P\left(\zeta_{\nu}\right)=\lim _{z \rightarrow \zeta_{\nu}} \frac{Q\left(z^{2}\right)}{2 Q(z)}=\zeta_{\nu} \frac{Q^{\prime}\left(\zeta_{\nu-1}\right)}{Q^{\prime}\left(\zeta_{\nu}\right)}
$$

for $\nu=1, \ldots, m$ with $\zeta_{0}=\zeta_{m}$, and therefore

$$
\prod_{\nu=1}^{m} P\left(\zeta_{\nu}\right)=\zeta_{m}^{1+2+\ldots+2^{m-1}}=\zeta_{m}^{2^{m}-1}=1
$$

in view of $\zeta_{m}^{2^{m}}=\zeta_{m}$. Hence, the solutions of (4) are $1, \epsilon, \ldots \epsilon^{m-1}$ with $\epsilon=e^{2 \pi i / m}$. This case appears e.g. for $Q(z)=(z-1) \prod_{\nu=1}^{m}\left(z-\zeta_{\nu}\right), \zeta_{\nu} \neq 1$, and $P(z)=\frac{1}{2}(1+z) \prod_{\nu=1}^{m}\left(z-\omega_{\nu}\right)$. If $\zeta_{1}, \ldots, \zeta_{m}$ are $(r+1)$-fold zeros of $Q(z)$, then, analogously,

$$
P\left(\zeta_{\nu}\right)=2^{r} \zeta_{\nu}^{r+1} \frac{Q^{(r+1)}\left(\zeta_{\nu-1}\right)}{Q^{(r+1)}\left(\zeta_{\nu}\right)}
$$

for $\nu=1, \ldots, m$ with $\zeta_{0}=\zeta_{m}$, and according to (4) it follows that $\lambda^{m}=2$. Hence, we have $\lambda=2^{r} \varepsilon^{\nu}$ for $\nu=0,1, \ldots, m-1$.

In the case $m=1$, which is only possible for $\zeta_{1}=1$, we have, without additional assumptions on the structure of $P(z)$, simply $\lambda=P(1)$.

3. If $\mathcal{M}_{2} \neq \emptyset$, then the vectors $\left(\zeta_{\nu}^{j}\right)_{j \geq 0}^{\mathrm{T}}$ with $\nu \in \mathcal{J}_{2}$, are eigenvectors of $\boldsymbol{A}$ to the eigenvalue 0 (see [1], Remark 2 in Section 4).

4. If $2^{m}-1$ is not a prime number, then the primitive period of the components $(5)$ can be a proper divisor of it as Example 2 shows with $m=4$ and $2^{4}-1=3 \cdot 5$.

Example 4. Let $P(z)=\frac{1}{2}(z+1)\left(z^{3}+1\right)^{2}=(z+1)^{3}\left(z^{2}-z+1\right)^{2}$ be the symbol of a two-slanted matrix $A$, so that $n=7$ and $Q(z)=(z-1)\left(z^{3}-1\right)^{2}=(z-1)^{3}\left(z^{2}+z+1\right)^{2}$ in the representation (14). To the threefold zero $\zeta=1$ of $Q(z)$ belong the eigenvalues $\lambda_{1}=4, \lambda_{2}=2, \lambda_{3}=1$ of $A$, further, we have the exceptional eigenvalue $\lambda_{4}=1$, and to the cycle of double zeros $\zeta_{1}=e^{2 \pi i / 3}, \zeta_{2}=e^{4 \pi i / 3}$ belong the eigenvalues $\lambda_{5}=2, \lambda_{6}=-2, \lambda_{7}=1$ and $\lambda_{8}=-1$. Corresponding to the theory, we find:

\begin{tabular}{r|r|l|l}
$\nu$ & $\lambda_{\nu}$ & \multicolumn{1}{|c}{ eigenvectors $w_{\nu}^{\mathrm{T}}$ of $A$} & components \\
\hline 1 & 4 & $(1,1,1,1,1,1,1,1)$ & constant \\
2 & 2 & $(7,5,3,1,-1,-3,-5,-7)$ & linear \\
3 & 1 & $(16,7,1,-2,-2,1,7,16)$ & quadratic \\
4 & 1 & $(1,0,0,0,0,0,0,0)$ & $0^{j}$ \\
5 & 2 & $(1,-1,0,1,-1,0,1,-1)$ & 3 -periodic \\
6 & -2 & $(1,1,-2,1,1,-2,1,1)$ & 3 -periodic \\
7 & 1 & $(-11,7,1,-2,-2,1,7,-11)$ & $w_{3 j}=-11+9 j, w_{3 j+1}=7-9 j, w_{3 j+2}=1$ \\
8 & -1 & $(1,1,-1,0,0,1,-1,-1)$ & $w_{3 j}=w_{3 j+1}=1-j, w_{3 j+2}=-1+2 j$
\end{tabular}

The components of the last two eigenvectors can be represented in the form (5) with 3periodic $\zeta_{k}^{j}$ and with linear $d_{k}(j)$. In analogy to $w_{4}^{\mathrm{T}}$, the eigenvalue 1 has also the eigenvector $(0,0,0,0,0,0,0,1)=\frac{1}{27}\left(w_{3}^{\mathrm{T}}-w_{7}^{\mathrm{T}}\right)-w_{4}^{\mathrm{T}}$. The zeros $\epsilon_{\nu}$ of $P(z)$ are $-1, e^{\pi i / 3}, e^{5 \pi i / 3}$. 
Example 5. Let $P(z)=\frac{1}{2}(z+1)\left(z^{3}-\frac{1}{2}(1-i \sqrt{7}) z^{2}-\frac{1}{2}(1+i \sqrt{7}) z+1\right)$ be the symbol of $A$, so that $n=4$ and $Q(z)=(z-1)\left(z^{3}+\frac{1}{2}(1-i \sqrt{7}) z^{2}-\frac{1}{2}(1+i \sqrt{7}) z-1\right)$ in (14). The zeros $\zeta_{\nu}$ of $Q(z)$ are $1, e^{2 \pi i / 7}, e^{4 \pi i / 7}, e^{8 \pi i / 7}$, hence the zeros $\varepsilon_{\nu}$ of $P(z)$ are $-1, e^{\pi i / 7}, e^{9 \pi i / 7}, e^{11 \pi i / 7}$. There appear two orbits without preperiods and with cycles of length $m=1$ resp. $m=3$. The corresponding matrix $A$ has the threefold eigenvalue 1 with the constant eigenvector $w_{1}^{\mathrm{T}}=(1,1,1,1,1)$, the exceptional eigenvector $w_{2}^{\mathrm{T}}$ and $w_{3}^{\mathrm{T}}=(0,0,0,0,1)$. Further, we obtain the eigenvalues $\lambda_{4}=-\frac{1}{2}(1-i \sqrt{3}), \lambda_{5}=\overline{\lambda_{4}}$, and the corressponding left eigenvectors of $A$ are restrictions of the 7 -periodic eigenvector

$$
\boldsymbol{w}_{4}^{\mathrm{T}}=(4,2+2 i \sqrt{7},-4+2 i a,-(\sqrt{7}+i) a,-2 i a,(\sqrt{3}-i) a, 2+2 i \sqrt{3}, 4, \ldots)
$$

of $\boldsymbol{A}$ with $a=\sqrt{3}+\sqrt{7}$ and of an analogous vector $\boldsymbol{w}_{5}^{\mathrm{T}}$ which is obtained by replacing $\sqrt{3}$ by $-\sqrt{3}$, also in $a$. The linear combination $\boldsymbol{w}_{3}^{\mathrm{T}}-\boldsymbol{w}_{2}^{\mathrm{T}}$ is also 7 -periodic, namely

$$
\boldsymbol{w}_{3}^{\mathrm{T}}-\boldsymbol{w}_{2}^{\mathrm{T}}=\left(-1,0,0,0,1, \frac{1}{2}(1+i \sqrt{7}), \frac{1}{2}(-1+i \sqrt{7}),-1, \ldots\right) .
$$

This example shows that the period $2^{m}-1$ can be greater than $n$.

\section{Root vectors of $A$}

In this section, we shall show that nonzero eigenvalues of a two-slanted matrix $A$ can never have proper root vectors with components of the form (5) and $\zeta_{k} \in \mathcal{M}_{P}$, where $P$ is the symbol (2) of $A$. From [1], Theorem 4.2, we know that the eigenvalue 0 can have proper root vectors.

Theorem 2 Let $\mu$ be a nonzero eigenvalue of $A$, and let all corresponding left eigenvectors $w$ possess components of the type (5) with $\zeta_{k} \in \mathcal{M}_{P}$ only. Then $A$ cannot have a root vector of height greater than 1 with components of the type (5).

Proof. Again, we carry out the proof for $\boldsymbol{A}$ instead of $A$. A vector $\tilde{\boldsymbol{w}}$ is a root vector of height 2 belonging to the eigenvalue $\mu$, if

$$
\tilde{\boldsymbol{w}}^{\mathrm{T}} \boldsymbol{A}=\mu \tilde{\boldsymbol{w}}^{\mathrm{T}}+\boldsymbol{w}^{\mathrm{T}}
$$

where $\boldsymbol{w}$ is an eigenvector of $\boldsymbol{A}$ to the same eigenvalue. According to our assumptions, the components of $\boldsymbol{w}$ have the form (5), and for the corresponding set $\mathcal{M}$, we first assume that it consists of one single orbit only. For the components of $\tilde{\boldsymbol{w}}$, we can use the ansatz

$$
\tilde{w}_{j}=\sum_{k=1}^{l} \tilde{d}_{k}(j) \zeta_{k}^{j}
$$

with the same $\zeta_{k}$ as in the representation of $\boldsymbol{w}$, because (15) is an inhomogeneous system, and we only need a special solution of it, so that all $\zeta_{k}$, which are not contained in the representation of $\boldsymbol{w}$, can be dropped in (16). The eigenvalue $\mu$ of $\boldsymbol{A}$ can be written in the 
form $\mu=\lambda / 2^{s}$ with $\lambda$ from (4) and $0 \leq s \leq r$, consequently, the polynomials $d_{k}(j)$ have at most the degree $s$. We write the polynomials $\tilde{d}_{k}(j)$ in (16) in the form

$$
\tilde{d}_{k}(j)=\sum_{\varrho=0}^{\gamma} \tilde{\alpha}_{k \varrho}\left(\begin{array}{l}
j \\
\varrho
\end{array}\right)
$$

for a certain $\gamma \in \mathbb{N}_{0}$. Hence, we obtain analogously as in (12)

$$
\left(\tilde{\boldsymbol{w}}^{\mathrm{T}} \boldsymbol{A}\right)_{j}=\frac{j^{\gamma}}{2^{\gamma} \gamma !}\left(\sum_{\nu \in \mathcal{J}_{1}} \tilde{\alpha}_{\nu \gamma} P\left(\zeta_{\nu+1}\right) \zeta_{\nu+1}^{j}+\sum_{\nu \in \mathcal{J}_{2}} \tilde{\alpha}_{\nu \gamma} P\left(\zeta_{n_{\nu}}\right) \zeta_{n_{\nu}}^{j}\right)+\ldots
$$

with index sets $\mathcal{J}_{1}, \mathcal{J}_{2}$ as in (7), and where the dots indicate terms with lower powers of $j$ (or zero for $\gamma=0$ ). On the other side,

$$
\frac{\lambda}{2^{s}} \tilde{w}_{j}=\frac{\lambda j^{\gamma}}{2^{s} \gamma !} \sum_{k=1}^{l} \tilde{\alpha}_{k \gamma} \zeta_{k}^{j}+\ldots, \quad w_{j}=\frac{j^{s}}{s !} \sum_{k=1}^{l} \alpha_{k s} \zeta_{k}^{j}+\ldots
$$

The equation $\tilde{\boldsymbol{w}}^{\mathrm{T}} \boldsymbol{A}=\frac{\lambda}{2^{s}} \tilde{\boldsymbol{w}}^{\mathrm{T}}+\boldsymbol{w}^{\mathrm{T}}$ can only be satisfied, if $\gamma \geq s$. In the case $\gamma>s$, we find by comparison of the coefficients of $j^{\gamma} /\left(2^{\gamma} \gamma\right.$ !) that

$$
\frac{\lambda}{2^{s-\gamma}} \sum_{k=1}^{l} \tilde{\alpha}_{k \gamma} \zeta_{k}^{j}=\sum_{\nu \in \mathcal{J}_{1}} \tilde{\alpha}_{\nu \gamma} P\left(\zeta_{\nu+1}\right) \zeta_{\nu+1}^{j}+\sum_{\nu \in \mathcal{J}_{2}} \tilde{\alpha}_{\nu \gamma} P\left(\zeta_{n_{\nu}}\right) \zeta_{n_{\nu}}^{j} .
$$

Analogously to (13), the determinant of the matrix of the linear system for the calculation of $\tilde{\alpha}_{k \gamma}$ reads

$$
\frac{\lambda^{l}}{2^{(s-\gamma) l}}\left(1-2^{(s-\gamma) m}\right)
$$

and is different from zero, so that the system has only the trivial solution.

In the case $\gamma=s$, we obtain analogously

$$
\lambda \sum_{k=1}^{l} \tilde{\alpha}_{k s} \zeta_{k}^{j}+2^{s} \sum_{k=1}^{l} \alpha_{k s} \zeta_{k}^{j}=\sum_{\nu \in \mathcal{J}_{1}} \tilde{\alpha}_{\nu s} P\left(\zeta_{\nu+1}\right) \zeta_{\nu+1}^{j}+\sum_{\nu \in \mathcal{J}_{2}} \tilde{\alpha}_{\nu s} P\left(\zeta_{n_{\nu}}\right) \zeta_{n_{\nu}}^{j} .
$$

Comparing coefficients, we obtain similar as in the foregoing section a system with the first $m$ equations

$$
B\left(\tilde{\alpha}_{k s}\right)=-2^{s}\left(\alpha_{k s}\right)
$$

with $B$ as in $(9)$ and vectors $\left(\alpha_{k s}\right)=\left(\alpha_{1 s}, \ldots, \alpha_{m s}\right)^{\mathrm{T}},\left(\tilde{\alpha}_{k s}\right)=\left(\tilde{\alpha}_{1 s}, \ldots, \tilde{\alpha}_{m s}\right)^{\mathrm{T}}$. The matrix $B$ has the left zero eigenvector

$$
v^{\mathrm{T}}=\left(\prod_{\nu=2}^{m} P\left(\zeta_{\nu}\right), \lambda \prod_{\nu=3}^{m} P\left(\zeta_{\nu}\right), \ldots, \lambda^{m-2} P\left(\zeta_{m}\right), \lambda^{m-1}\right),
$$


and in view of $\alpha_{k s}=d_{k}$ and (11), cf. the proof of Theorem 1, we have the scalar product

$$
v^{\mathrm{T}} \cdot\left(\alpha_{k s}\right)=m \lambda^{m-1},
$$

which is different from zero. Hence (17) is unsolvable, so that there cannot exist a root vector of height 2 with components (16) belonging to one single orbit of $\mathcal{M}_{P}$. But the case of several orbits is also impossible, since we can do our considerations for each orbit separately. Moreover, since there are no root vectors of height 2 with the components (16) there also cannot exist root vectors of a greater height with such components, and the theorem is proved.

\section{Application to two-scale difference equations}

In the last part of the paper, we want to apply the above results to two-scale difference equations of the form (3). As shown in [5], (3) can only have a nontrivial compactly supported (distributional) solution $\varphi$ if $P(1)=2^{k}$ for some nonnegative integer $k$. In this case we find $\operatorname{supp} \varphi=[0, n]$. For simplicity, we mainly use the following assumptions on the symbol $P$ in $(2)$ :

(A1) $P(1)=1$.

(A2) $\left|c_{0}\right|<1,\left|c_{1}\right|<1$.

The assumption (A1) is not restrictive, since each solution of (3) whose symbol $P$ satisfies $P(1)=2^{m}$ can be seen as an $m$ th (distributional) derivative of a solution of (3) with symbol $2^{-m} P$. Further, (A2) is a necessary condition for continuity of $\varphi$.

Introducing the vector $\psi(t)=(\varphi(t), \ldots \varphi(t+n))^{\mathrm{T}}$, equation $(3)$ can be rewritten in the form

$$
\psi\left(\frac{t}{2}\right)=A \psi(t) \quad \text { for }-1 \leq t \leq 1
$$

with $A$ from (1). Equivalently, we find $\boldsymbol{\psi}\left(\frac{t}{2}\right)=\boldsymbol{A} \boldsymbol{\psi}(t)$ for $-\infty<t \leq 1$ with $\boldsymbol{\psi}(t)=$ $(\varphi(t), \varphi(t+1), \ldots)^{\mathrm{T}}$. In order to study the properties of $\varphi$, in [1] the space

$$
\mathcal{L}=\operatorname{span}\left\{\boldsymbol{w}=\left(w_{j}\right)_{j \geq 0}: \boldsymbol{w}^{\mathrm{T}} \boldsymbol{\psi}(t)=c, t \in(-\infty, 1] \text { a.e. }\right\}
$$

has been considered. In particular, we have (see [1]):

Proposition 1 Let $\varphi$ be a nontrivial, compactly supported, integrable solution of (3), and let (A1), (A2) be satisfied. Then we have:

(i) The vector $\boldsymbol{e}=(1,1, \ldots)^{\mathrm{T}}$ is contained in $\mathcal{L}$.

(ii) If $\boldsymbol{w} \in \mathcal{L}$, then $\left(\boldsymbol{w}^{\mathrm{T}} \boldsymbol{A}\right)^{\mathrm{T}} \in \mathcal{L}$.

(iii) Let the solution $\varphi$ be bounded in neighbourhoods of the points $k(k=0, \ldots, n)$. If $\lambda \in \mathbb{C}$ with $|\lambda|>1$ is an eigenvalue of $\boldsymbol{A}$, then the corresponding left eigenvectors of $\boldsymbol{A}$ are contained in $\mathcal{L}$.

(iv) Let $\varphi$ be continuous in the points $k(k=0, \ldots, n)$. Then the left eigenvectors of $\boldsymbol{A}$ 
corresponding to an eigenvalue $\lambda$ with $|\lambda|=1$ are contained in $\mathcal{L}$.

(v) If $\boldsymbol{w}=\left(w_{j}\right)_{j \geq 0} \in \mathcal{L}$. Then $w_{j}$ has the form

$$
w_{j}=\sum_{k=1}^{l} d_{k}(j) \zeta_{k}^{j} \quad(j \geq 0)
$$

with $l<n$, where $\zeta_{k} \in \mathbb{C} \backslash\{0\}$ are zeros of the polynomial

$$
B(z)=\sum_{\mu=0}^{n-1}\left(\varphi\left(\mu+t_{1}\right)-\varphi(\mu)\right) z^{\mu}
$$

for each choice of $t_{1} \in(0,1)$. The coefficients $d_{k}(j)$ are polynomials in $j$ of degree $\nu_{k}$, if $\zeta_{k}$ is a zero of $B(z)$ of multiplicity $\nu_{k}+1$.

(vi) If $\boldsymbol{w} \in \mathcal{L}$ with representation (19) is an eigenvector of $\boldsymbol{A}$ then the numbers $\zeta_{k}$ occuring in (19) are roots of unity and form a closed set under the mapping $z \mapsto z^{2}$.

Let $\mathcal{M}^{B}$ be the largest set of zeros of $B(z)$ (for each choice of $t_{1}$ ) being closed under the mapping $z \mapsto z^{2}$. Then, under the assumptions of Proposition 1 , it follows that $\mathcal{M}^{B}=\mathcal{M}_{P}$, where $P(z)$ is the symbol (2) of $A$ and $\mathcal{M}_{P}$ is defined as at the end of Section 2. This assertion directly follows from Proposition 1 and Theorem 3.1 in [1].

Summarizing and simplifying the above results and observing that the $r$-th (distributional) derivative of $\boldsymbol{\psi}(t)$ satisfies $\boldsymbol{\psi}^{(r)}\left(\frac{t}{2}\right)=2^{r} \boldsymbol{A} \boldsymbol{\psi}(t)$, we obtain:

Theorem 3 Let $\varphi$ be a nontrivial compactly supported (distributional) solution of (3). Then the components of an eigenvector of $A$ to the eigenvalue $\mu$ have the form (5), if one of the following conditions is satisfied:

(i) $\varphi(t)$ is integrable, bounded and $|\mu|>1$,

(ii) $\varphi(t)$ is continuous and $|\mu| \geq 1$,

(iii) the $r$-th derivative of $\varphi(t)$ is bounded and $|\mu|>\frac{1}{2^{r}}$,

(iv) the $r$-th derivative of $\varphi(t)$ is continuous and $|\mu| \geq \frac{1}{2^{r}}$.

Applying Theorem 2, we find the following generalization of Theorem 4.1 in [1].

Corollary 2 If one of the conditions of Theorem 3 is satisfied, then to the eigenvalue $\mu$ of A there exist no root vectors of height greater than 1 .

Remark. The solution of (3) has in the first, resp. last subinterval of its support the structure

$$
\begin{aligned}
& \varphi(t)=t^{\alpha} R\left(\frac{\ln t}{\ln 2}\right) \quad \text { for } 0 \leq t \leq 1, \\
& \varphi(t)=(n-t)^{\beta} S\left(\frac{\ln (n-t)}{\ln 2}\right) \quad \text { for } n-1 \leq t \leq n
\end{aligned}
$$

with $2^{-\alpha}=c_{0}, 2^{-\beta}=c_{n}$ and 1-periodic functions $R(x)=R(x-1), S(x)=S(x-1)$. If we look for real solutions only, then in case of $c_{0}<0$ we can choose $2^{-\alpha}=\left|c_{0}\right|$ and $R(x)=-R(x-1)$ antiperiodic. For $c_{n}<0$, the situation is analogous. These representations of $\varphi(t)$ are useful for a discussion of the behaviour of $\varphi$. 


\section{The start vector $\psi(0)$}

From (18) it follows for nontrivial $\varphi$ that $\psi(0)$ is a right eigenvector of $A$ to the eigenvalue 1. If $\psi(0)$ is known, then (18) implies an iterative algorithm for the computation of $\varphi\left(2^{-j} l\right)$, $l=0, \ldots, 2^{j} n$, and for continuous $\varphi$, the interpolation splines

$$
\varphi_{j}(t)=\sum_{l=0}^{2^{j} n} \varphi\left(2^{-j} l\right) h\left(2^{j} t-l\right)
$$

with the hat function $h(t)=(1-|t|) \chi_{[-1,1]}(t)$ uniformly converge to $\varphi$ for $j \rightarrow \infty$, see $[2,5,11]$. (Here, $\chi_{[-1,1]}$ denotes the characteristic function of $[-1,1]$.) However, if the eigenvalue 1 of $A$ is not simple, then the computation of $\psi(0)$ is more delicate.

Using the results of Theorem 1 and Theorem 3 , we observe that multiple eigenvalues 1 can only occur if the set $\mathcal{M}_{P}$ contains a corresponding number of different orbits. In the following, we want to present three different methods to determine $\psi(0)$.

Theorem 4 Let (3) possess a nontrivial compactly supported, continuous solution. Then the right eigenvector $\psi(0)$ of $A$ to the eigenvalue 1 is determined by the condition $w^{\mathrm{T}} \psi(0)=0$ for all left eigenvectors $w^{\mathrm{T}}$ of $A$ to the eigenvalue 1 with components of the form (5) with $\zeta_{k} \in \mathcal{M}_{P} \backslash\{1\}$.

Proof. According to (ii) from Theorem 3, the components of all left eigenvectors $w^{\mathrm{T}}$ of $A$ to the eigenvalue 1 have the form (5), and Proposition 1 implies $w^{\mathrm{T}} \psi(t)=w^{\mathrm{T}} \psi(0)$ for $-1 \leq t \leq 1$. Moreover, we have according to [1], Theorem 2.4,

$$
\sum_{j=0}^{n} j^{\nu} \zeta_{k}^{j} \varphi(t+j)=c_{\nu k}
$$

for $-1 \leq t \leq 1$ and $0 \leq \nu \leq \nu_{k}$, where $\nu_{k}$ is the degree of the coefficient $d_{k}(j)$ in $(5)$. In view of $\varphi(t)=0$ for $t \notin(0, n)$, it follows for $0 \leq t \leq 1$ that

$$
\sum_{j=0}^{n-1} j^{\nu} \zeta_{k}^{j} \varphi(t+j)=c_{\nu k}
$$

and after replacing $t$ by $t-1$,

$$
c_{\nu k}=\sum_{j=1}^{n} j^{\nu} \zeta_{k}^{j} \varphi(t-1+j)=\sum_{j=0}^{n-1}(j+1)^{\nu} \zeta_{k}^{j+1} \varphi(t+j)
$$

also for $0 \leq t \leq 1$. For $\nu=0$, this means $c_{0 k}=\zeta_{k} c_{0 k}$, i.e. $c_{0 k}=0$ for $\zeta_{k} \neq 1$. If we have proved already $c_{\mu k}=0$ for $0 \leq \mu<\nu \leq \nu_{k}$, we find $c_{\nu k}=\zeta_{k} c_{\nu k}$ and therefore $c_{\nu k}=0$ for $\zeta_{k} \neq 1$ and all integers $\nu \in\left[0, \nu_{k}\right]$. Hence, we have $w^{\mathrm{T}} \psi(t)=0$ for all left eigenvectors with components of the form (5) with $\zeta_{k} \neq 1$ for all $k$, so that, for $t=0$, the theorem is proved.

As a consequence, we can determine $\psi(0)$ in the following way: Let $W_{1}^{\mathrm{T}}$ be the matrix of all left eigenvectors of $A$ to the eigenvalue 1 . We can choose the components of the eigenvectors 
according to (5) with sets $\mathcal{M} \subset \mathcal{M}_{P}$, consisting of one orbit only. According to Theorem 3.3 in [1], we have always one eigenvector with $\zeta_{1}=1$. We choose the components of this vector as the first row of $W_{1}^{\mathrm{T}}$. Let $U_{1}$ be the matrix of all right eigenvectors of $A$ to 1 , such that $W_{1}^{\mathrm{T}} U_{1}=I$. Then the vector of the first column of $U_{1}$ is $\psi(0)$ (up to a constant factor).

Example 6. Consider the two-scale difference equation corresponding to the symbol $P(z)=$ $\frac{1}{4}\left(z^{3}+1\right)^{2}\left(z^{5}+1\right)^{2}$. The zero set of $P(z)$ is $\left\{-1, e^{\pi i / 3}, e^{-\pi i / 3}, e^{\pi i / 5}, e^{-\pi i / 5}, e^{3 \pi i / 5}, e^{-3 \pi i / 5}\right\}$. Hence, we find $\mathcal{M}_{P}=\left\{1, e^{2 \pi i / 3}, e^{4 \pi i / 3}, e^{2 \pi i / 5}, e^{4 \pi i / 5}, e^{8 \pi i / 5}, e^{6 \pi i / 5}\right\}$. The set $\mathcal{M}_{P}$ contains 3 different orbits, namely $\{1\},\left\{e^{2 \pi i / 3}, e^{4 \pi i / 3}\right\}$ and $\left\{e^{2 \pi i / 5}, e^{4 \pi i / 5}, e^{8 \pi i / 5}, e^{6 \pi i / 5}\right\}$. Indeed, the corresponding matrix $A$ possesses the eigenvalue 1 with multiplicity 3 . The two left eigenvectors of $A$ of the form (5) with $\zeta_{k}$ from the second and from the third subset, respectively, satisfy $w^{\mathrm{T}} \psi(0)=0$ (see [2], Example 2.6 (ii)). Note that here we have $P(1)=4$.

In order to explain the second possibility to determine $\psi(0)$, we modify Proposition 3.2 of [1]:

Proposition 2 Let $\varphi(t)$ be a nontrivial compactly supported solution of (3), and let the corresponding refinement mask have the splitting

$$
P(z)=\frac{q\left(z^{2}\right)}{q(z)} \tilde{P}(z)
$$

with polynomials $q(z), \tilde{P}(z)$ and $q(0) q(1) \neq 0$, say

$$
q(z)=\sum_{k=0}^{m} q_{k} z^{k} .
$$

Then the equation (3) with the symbol $\tilde{P}(z)$ has a compactly supported solution $\tilde{\varphi}(t)$ and

$$
\varphi(t)=\sum_{k=0}^{m} q_{k} \tilde{\varphi}(t-k) .
$$

Let now $A$ be the two-slanted matrix (1) corresponding to (3) with a nontrivial compactly supported continuous solution $\varphi$, and let 1 be a multiple eigenvalue of $A$. Then $\mathcal{M}_{P}$ consists of several orbits $\mathcal{M}^{1}, \ldots, \mathcal{M}^{\kappa}$ with $\kappa>1$. Let $\mathcal{M}^{1}$ be the orbit of $\mathcal{M}_{P}$ containing $\zeta=1$, and $\mathcal{M}^{0}=\mathcal{M}_{P} \backslash \mathcal{M}^{1}=\left\{\zeta_{k}, \ldots, \zeta_{l}\right\}$ with a certain $k>1$. Let $\omega_{k}, \ldots, \omega_{l}$ be the roots of the elements of $\mathcal{M}^{0}$ not belonging to $\mathcal{M}^{0}$. All these roots are zeros of $P$. Let $s_{\nu}+1$ be the multiplicity of the zero $\omega_{\nu}$ of $P, \nu=k, \ldots, l$, and $r^{\mu}=\min s_{\nu}$ for all $\nu$ with $\omega_{\nu}^{2} \in \mathcal{M}^{\mu}$, $\mu=2, \ldots, \kappa$. For these indices $\nu$, we also use the notation $r_{\nu}=r^{\mu}$. Now, we choose $q(z)=\prod_{\nu=k}^{l}\left(z-\zeta_{\nu}\right)^{r_{\nu}}$, so that $q\left(z^{2}\right) / q(z)=\prod_{\nu=k}^{l}\left(z-\omega_{\nu}\right)^{r_{\nu}}$. According to (20), the set $\mathcal{M}_{\tilde{P}}$ can only consist of one single orbit, and the two-slanted matrix $\tilde{A}$ belonging to $\tilde{P}(z)$ must have 1 as a simple eigenvalue. We choose $q_{k}=0$ for $k \notin\{0,1, \ldots, m\}$ and introduce the rectangular Toeplitz matrix $Q=\left(q_{j-k}\right), j=0,1, \ldots, n$ and $k=0,1, \ldots, m$, as well as $\tilde{\psi}(t)=(\tilde{\varphi}(t), \tilde{\varphi}(t+1), \ldots, \tilde{\varphi}(t+m))^{\mathrm{T}}$. Hence, we can write $(21)$ as $\psi(t)=Q \tilde{\psi}(t)$, which implies $\psi(0)=Q \tilde{\psi}(0)$. Since $\tilde{\psi}(0)$ can be determined uniquely up to a constant factor, also the wanted $\psi(0)$ is fixed by the foregoing formula. 
Let us mention that (20) implies $Q \tilde{A}=A Q$.

The third possibility to determine $\psi(0)$ was already described in [2]. Let as before $W_{1}^{\mathrm{T}}$ be the matrix of all left eigenvectors of $A$ to the eigenvalue 1 , and $U_{1}$ the matrix of all corresponding right eigenvectors, such that $W_{1}^{\mathrm{T}} U_{1}=I$. We use the shifting matrix $V=\left(\delta_{j-k+1}\right), j, k=$ $0,1, \ldots, n$, where $\delta_{j}$ is the simplified Kronecker symbol, i.e. $\delta_{0}=1$ and $\delta_{j}=0$ for $j \neq 0$. It was shown in [2] by means of the ansatz $\psi(0)=U_{1} x$ that the vector $x$ satisfies the equations

$$
x=W_{1}^{\mathrm{T}} V^{\mathrm{T}} U_{1} x \text { and } x=W_{1}^{\mathrm{T}} V U_{1} x .
$$

Further, it was conjectered in [2] that $x$ is uniquely determined by these equations up to a constant factor. Anyway, if (22) has only the trivial solution, then (3) does not have a nontrivial compactly supported continuous solution.

Example 7. For real parameters $a, p$, we consider (3) with $n=5$ and $c_{0}=a, c_{1}=a p, c_{2}=$ $1-a-a p, c_{3}=(1+p)(1-a p), c_{4}=a p, c_{5}=p(a p-1)$, i.e., the two-scale symbol $P(z)$ reads

$$
P(z)=\frac{1}{2}(1+z)\left(a+a(p-1) z+(1-2 a p) z^{2}+p(a+1-a p) z^{3}+p(a p-1) z^{4}\right) .
$$

Observe that $P(1)=1$, hence (3) provides a (distributional) compactly supported solution $\varphi$. The corresponding matrix (1) has 1 as a double eigenvalue with the matrix of right eigenvectors

$$
U_{1}=\left[\begin{array}{cr}
0 & 0 \\
a\left(p^{2}+p+1\right) & 0 \\
(1-a p)\left(p^{2}+p+1\right) & 0 \\
0 & 1 \\
0 & -p \\
0 & 0
\end{array}\right] .
$$

The factor $p^{2}+p+1$ in the first column was choosen in order to simplify later formulas. The matrix $W_{1}^{\mathrm{T}}$ of the corresponding left eigenvectors with $W_{1}^{\mathrm{T}} U_{1}=I$ was determined by means of DERIVE and is too complicated to write it down here. In general, the two equations (22) have linearly independent solutions $x$, so that (3) does not have a nontrivial, compactly supported, continuous solution. However, in the cases

$$
a=\frac{1}{p}, a=-\frac{1}{p}, a=\frac{p+1}{p^{2}+p+1},
$$

(22) has a common nontrivial solution $x$. For $a=\frac{1}{p}$, we have $c_{5}=0$, so that $n$ reduces to $n=4$ and $c_{4}=1$. Hence the solution $\varphi$ of (3) is not continuous (see e.g. [3]). For $a=-\frac{1}{p}$, we have $c_{0}=\frac{1}{p}, c_{5}=-2 p$, and therefore for every $p$ once more a contradiction to the necessary condition (A2) for the existence of the wanted continuous solution. In the last case of (24), we find $x=(1,1)^{\mathrm{T}}$ and

$$
W_{1}^{\mathrm{T}}=\frac{1}{2\left(p^{2}+p+1\right)}\left[\begin{array}{llllll}
p(p+1) & p+1 & p^{2}+1 & p(p+1) & p+1 & p^{2}+1 \\
p^{2}+2 p+2 & -p & p(p+1) & p^{2}+2 p+2 & -p & p(p+1)
\end{array}\right]
$$



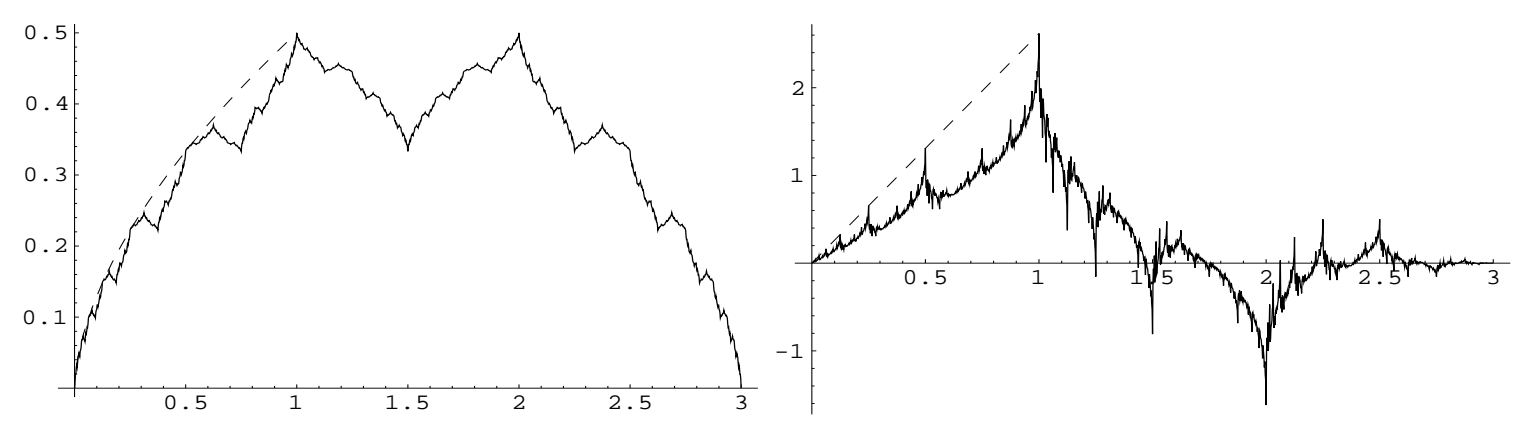

Figure 2: Solution $\tilde{\varphi}(t)$ for $p=-0.5$ and, for $t \in[0,1], \tilde{\varphi}(1) t^{0.5849625}$ (dashed).

Figure 3: Solution $\tilde{\varphi}(t)$ for $p=(\sqrt{5}+1) / 2$ and, for $t \in[0,1], \tilde{\varphi}(1) t$ (dashed).

so that $\psi(0)=(0, p+1,1,1,-p, 0)^{\mathrm{T}}$. The two-scale symbol allows a further factorization, namely

$$
2\left(p^{2}+p+1\right) P(z)=(1+z)\left(1-z+z^{2}\right)\left(p+1+p(p+1) z-p z^{2}\right) .
$$

Corresponding to the theory, we have a constant left eigenvector $(p+2,1-p) W_{1}^{\mathrm{T}}$ and a $3-$ periodic one to the eigenvalue 1 , and a 3 -periodic left eigenvector $(p, 1,-p-1, p, 1,-p-1)$ to the eigenvalue -1 . Besides of the already mentioned eigenvalues as well as $c_{0}$ and $c_{5}$, we also obtain the eigenvalue $\frac{p(p+1)}{p^{2}+p+1}$. The points $p=0$ and $p=-1$ must be excluded, since they imply $c_{0}=1$ and $c_{5}=1$, respectively.

Observe that the computation of $\varphi$ can be simplified by means of Proposition 2,

$$
\varphi(t)=\tilde{\varphi}(t)+\tilde{\varphi}(t-1)+\tilde{\varphi}(t-2)
$$

where $\tilde{\varphi}$ itself is a solution of (3) with symbol

$$
\tilde{P}(z)=\frac{(1+z)\left(p+1+p(p+1) z-p z^{2}\right)}{2\left(p^{2}+p+1\right)}=\frac{1+z}{2} Q(z),
$$

where $Q(z)=\frac{1}{2}\left(q_{0}+q_{1} z+q_{2} z^{2}\right)=\left(p+1+p(p+1) z-p z^{2}\right) /\left(p^{2}+p+1\right)$.

As already remarked at the end of Section 5, in the neighborhood of 0 , the function $\tilde{\varphi}$ has a similar behaviour as $\tilde{\varphi}(1) t^{\alpha}$ with $\alpha=-\log _{2}\left|c_{0}\right|=\log _{2}\left|\frac{p+1}{p^{2}+p+1}\right|$ (see Figures 2 and 3 ). Analogously, $\tilde{\varphi}(t)$ behaves as $\tilde{\varphi}(n-1)(n-t)^{\beta}$ in the neighborhood of $n$ with $\beta=-\log _{2}\left|c_{n}\right|=$ $\log _{2}\left|\frac{p}{p^{2}+p+1}\right|$. Further, a negative $c_{0}$ (resp. $c_{n}$ ) causes changes of sign of the solution $\tilde{\varphi}$ in $(0,1)$ (resp. $(n-1, n)$ ). For $p=-0.5$ we obtain $c_{0}=c_{3}=2 / 3$ and $\alpha=\beta=0.5849625$ (see Figure $2)$. For $p=(\sqrt{5}+1) / 2$ it follows that $c_{0}=1 / 2, c_{3}=(1-\sqrt{5}) / 4$ and $\alpha=1, \beta=1.6942419$ (see Figure 3).

Finally, we want to estimate the Sobolev exponent

$$
s(\varphi)=s(\tilde{\varphi})=\sup \left\{\delta: \int_{-\infty}^{\infty}|\hat{\varphi}(u)|^{2}\left(1+|u|^{2}\right)^{\delta} d u<+\infty\right\}
$$

of $\tilde{\varphi}$ (resp. $\varphi$ ) using the transfer operator $T: L^{2}([0,2 \pi]) \mapsto L^{2}([0,2 \pi])$ corresponding to $Q(z)$. This operator is defined by

$$
T H(2 u)=\left|Q\left(e^{-i u}\right)\right|^{2} H(u)+\left|Q\left(-e^{-i u}\right)\right|^{2} H(u+\pi), \quad H \in L^{2}([0,2 \pi]) .
$$


It can be simply observed that $T$ is invariant on the set $\Pi_{N}$ of trigonometric polynomials of degree at most $N$ with $N \geq 2$. Considering the basis $\left\{e_{l}=e^{-i l .}: l=-N,-N+1, \ldots, N-\right.$ $1, N\}$ of $\Pi_{N}$ we find with $\mu=k+l$

$$
\begin{aligned}
\left(T e_{2 l}\right)(u) & =\left(\left|Q\left(e^{-i u / 2}\right)\right|^{2}+\left|Q\left(-e^{-i u / 2}\right)\right|^{2}\right) e^{-i u 2 l / 2} \\
& =\frac{1}{2} \sum_{m \in \mathbb{Z}} \sum_{k \in \mathbb{Z}} q_{m-2 k} q_{m} e^{-i u k} e^{-i u l} \\
& =\sum_{\mu \in \mathbb{Z}}\left(\frac{1}{2} \sum_{m \in \mathbb{Z}} q_{m-2 \mu+2 l} q_{m}\right) e_{\mu} .
\end{aligned}
$$

Analogously,

$$
\begin{aligned}
\left(T e_{2 l-1}\right)(u) & =\left(\left|Q\left(e^{-i u / 2}\right)\right|^{2}-\left|Q\left(-e^{-i u / 2}\right)\right|^{2}\right) e^{-i u(2 l-1) / 2} \\
& =\sum_{\mu \in \mathbb{Z}}\left(\frac{1}{2} \sum_{m \in \mathbb{Z}} q_{m-2 \mu+2 l-1} q_{m}\right) e_{\mu} .
\end{aligned}
$$

Since $Q\left(e^{-i u}\right) \in \Pi_{2}$, we only need to consider the spectral properties of $T$ restricted to $\Pi_{2}$. Taking the basis elements of $\Pi_{2}$ we have $\left(T e_{\nu}\right)_{\nu=-2}^{2}=\mathcal{T}\left(e_{\nu}\right)_{\nu=-2}^{2}$ with the representing matrix

$$
\begin{gathered}
\mathcal{T}=\frac{1}{2}\left(\sum_{m=0}^{2} q_{m-2 \mu+j} q_{m}\right)_{j, \mu=-2}^{2} \\
=\frac{2}{\left(p^{2}+p+1\right)^{2}}\left(\begin{array}{ccccc}
-p(p+1) & \left(p^{2}+p+1\right)^{2} & -p(p+1) & 0 & 0 \\
0 & p(p+1) & p(p+1) & 0 & 0 \\
0 & -p(p+1) & \left(p^{2}+p+1\right)^{2} & -p(p+1) & 0 \\
0 & 0 & p(p+1) & p(p+1) & 0 \\
0 & 0 & -p(p+1) & \left(p^{2}+p+1\right)^{2} & -p(p+1)
\end{array}\right) .
\end{gathered}
$$

The Sobolev exponent is now given by $s(\varphi)=1-\log _{4} \rho$, where $\rho$ denotes the spectral radius of $\mathcal{T}$ (cf. [7, 8]). For all $p \in \mathbb{R} \backslash\{0,-1\}$, the spectral radius of $\mathcal{T}$ is smaller then 2 , hence $s(\phi)>0.5$ and we obtain a continuous solution $\varphi$ of (3). For $p=-0.5$, the maximal regularity is found; in this case $\rho=1.267013804$ and $s(\varphi)=0.8292838787$ (see Figure 2). For $p=(\sqrt{5}+1) / 2$, we find $\rho=1.878299127$ and $s(\varphi)=0.5452865817$ (see Figure 3 ).

\section{References}

[1] L. Berg and G. Plonka, Compactly supported solutions of two-scale difference equations, Linear Algebra Appl., to appear.

[2] L. Berg and G. Plonka, Some notes on two-scale difference equations, Recent Progress in Functional Equations (T. M. Rassias, ed.), to appear.

[3] D. Colella and C. Heil, Characterizations of scaling functions, I. Continuous solutions, J. Math. Anal. Appl. 15 (1994), 496-518. 
[4] I. Daubechies, Ten Lectures on Wavelets, SIAM, Philadelphia, 1992.

[5] I. Daubechies and J. Lagarias, Two-scale difference equations: I. Global regularity of solutions, SIAM J. Math. Anal. 22 (1991), 1388-1410.

[6] I. Daubechies and J. Lagarias, Two-scale difference equations: II. Local regularity, infinite products of matrices and fractals, SIAM J. Math. Anal. 23 (1992), 1031-1079.

[7] T. Eirola, Sobolev characterization of solutions of dilation equations, SIAM J. Math. Anal. 23 (1992), 1015-1030.

[8] Q. Jiang, On the regularity of matrix refinable functions, SIAM J. Math. Anal., to appear.

[9] M. Kuczma, B. Choczewski and G. Ger, Iterative Functional Equations, Cambridge Univ. Press, Cambridge, 1990.

[10] W. Lawton, S. L. Lee and Z. Shen, Characterization of compactly supported refinable splines, Adv. Comput. Math. 3 (1995) 137-146.

[11] C. Micchelli and H. Prautzsch, Uniform refinement of curves, Linear Algebra Appl. 114/115 (1989), 841-870.

[12] G. Targonski, Topics in Iteration Theory, Vanderhoeck \& Ruprecht, Göttingen-Zürich, 1981.

\section{Adresses}

Lothar Berg

Fachbereich Mathematik

Universität Rostock

D-18051 Rostock

Germany
Gerlind Plonka

Fachbereich Mathematik

Universität Duisburg

D-47048 Duisburg

Germany 